\title{
Effects of Angiotensin II Generated by an Angiotensin Converting Enzyme-independent Pathway on Left Ventricular Performance in the Conscious Baboon
}

Brian D. Hoit, Yanfu Shao, Akio Kinoshita, * Marjorie Gabel, Ahsan Husain, ${ }^{\star}$ and Richard A. Walsh

Division of Cardiology, University of Cincinnati Medical Center, Cincinnati, Ohio 45267-0542; and *Department of Heart and Hypertension Research, The Cleveland Clinic, Cleveland, Ohio 44195-5069.

\begin{abstract}
Human chymase is a serine proteinase that converts angiotensin (Ang) I to Ang II independent of angiotensin converting enzyme (ACE) in vitro. The effects of chymase on systemic hemodynamics and left ventricular function in vivo were studied in nine conscious baboons instrumented with a $L V$ micromanometer and $L V$ minor axis and wall thickness sonomicrometer crystal pairs. Measurements were made at baseline and after [Pro ${ }^{11} D A a^{12}$ ] Ang I, a specific substrate for human chymase, was given in consecutive fashion as a $0.1 \mathrm{mg}$ bolus, an hour-long intravenous infusion of $5 \mathrm{mg}$, a $3 \mathrm{mg}$ bolus, and after $5 \mathrm{mg}$ of an Ang II receptor antagonist. [Pro ${ }^{11}$ DAla $^{12}$ ] Ang I significantly increased LV systolic and diastolic pressures, LV end-diastolic and end systolic dimensions and the time constant of $\mathrm{LV}$ relaxation and significantly decreased $L V$ fractional shortening and wall thickening. Administration of a specific Ang II receptor antagonist reversed all the hemodynamic changes. In separate studies, similar results were obtained in six of the baboons with ACE blockade (20 mg, intravenous captopril). Postmortem studies indicated that chymase-like activity was widely distributed in multiple tissues. Thus, in primates, Ang I is converted into Ang II by an enzyme with chymaselike activity. This study provides the first in vivo evidence of an ACE-independent pathway for Ang II production. ( $J$. Clin. Invest. 1995. 95:1519-1527.) Key words: human heart chyase • angiotensin II • converting enzyme - myocardial contractility $\cdot$ hemodynamics
\end{abstract}

\section{Introduction}

Angiotensin I-converting enzyme (EC. 3.4.15.1) (ACE) ${ }^{1}$ is a zinc metallopeptidase that converts angiotensin (Ang) I to the vasopressor hormone Ang II (1). ACE is present in plasma and in a number of tissues including blood vessels, kidney, heart,

Address correspondence to Brian D. Hoit, M.D., Division of Cardiology, University of Cincinnati Medical Center, PO Box 670542, Cincinnati, OH 45267-0542. Phone: 513-558-3070; FAX: 513-558-3116.

Received for publication 12 May 1994 and in revised form 14 November 1994.

1. Abbreviations used in this paper: ACE, angiotensin I-converting enzyme; Ang, angiotensin; LV, left ventricular.

J. Clin. Invest.

(C) The American Society for Clinical Investigation, Inc.

0021-9738/95/04/1519/09 \$2.00

Volume 95, April 1995, 1519-1527 brain, and the adrenal gland (2). ACE inhibitors are widely used in the treatment of hypertension and congestive heart failure. However, because Ang II is readily detected in plasma and tissues during ACE inhibitor therapy (3), some investigators have speculated that enzymes other than ACE are involved in the in vivo conversion of Ang I to Ang II. For example, while examining the mechanism of action of Ang $I$ in the dog aorta, Okunishi and co-workers (4) described both ACE-dependent and ACE-independent pathways of Ang II formation and indicated that a chymotrypsin-like enzyme was responsible for the Ang II formation that occurred in the presence of an ACE inhibitor. While studying the biochemical pathways of Ang II formation in the human heart, we observed that $>75 \%$ of the Ang II-forming enzyme activity in human left ventricular tissue homogenates was not blocked by ACE inhibitors, but was blocked by serine proteinase inhibitors (5). Our subsequent studies showed that the major Ang II-forming serine proteinase from the human cardiac ventricles was a chymase. Human chymase, isolated from the heart and skin, is a glycoprotein with an apparent molecular weight of $\sim 30 \mathrm{kD}$ by SDS-PAGE (6). The human chymase gene and cDNA have recently been cloned (7).

In situ hybridization and EM-immunocytochemical studies indicate that the mast cells and endothelial cells located within the human heart and its vessels are the site of chymase synthesis and storage (8). A high level of chymase-like immunoreactivity is also localized to the cardiac interstitium and is likely associated with the interstitial extracellular matrix. In endothelial cells, chymase-like immunoreactivity is present in Weibel-Palade bodies. Recent studies suggest that secretion of the von-Willebrand factor stored in Weibel-Palade bodies is polarized, with the bulk of the secretion occurring in the basolateral direction (9); this would indicate that chymase is also released basolaterally. Consistent with this conjecture, EM-immunocytochemical studies indicate the presence of chymase-like immunoreactivity in the basolateral region of endothelial cells (8). The immunocytochemical studies localizing chymase in the heart and vessels suggest that chymase plays a significant role in the interstitial formation of Ang II. However, since mast cells are a major site of elaboration of chymase and since procurement of tissues could easily lead to mast cell degranulation and release of chymase, the possibility exists that in vivo chymase-dependent Ang II formation may be much less significant than that inferred from immunocytochemical studies or studies using isolated tissues.

In vivo pharmacological studies with Ang I in the presence of ACE inhibitors are one way to show the importance of an alternative pathway to ACE in Ang II formation. However, in these studies the possibility exists that even in the presence of high concentrations of ACE inhibitor, tissue ACE is not fully inhibited or that Ang I has intrinsic activity which allows a 
direct response without conversion in Ang II (10). These studies also do not rule out the possibility that nonspecific carboxypeptidases rather than chymase can convert Ang I to Ang II.

In the present study we use a novel approach to show that chymase dependent Ang II formation can occur in conscious baboons. This approach uses a selective Ang II containing substrate, [Pro ${ }^{11} \mathrm{DAla}^{12}$ ] Ang I, which is an inactive precursor that yields Ang II upon incubation with chymase, but not ACE (11, 12). Extensive studies on the nature of the substrate binding site of human chymase has revealed key differences in the way human chymase binds Ang I prior to catalysis, compared to the interaction between ACE and Ang I. These differences were exploited to design [Pro ${ }^{11}$ DAla ${ }^{12}$ ] Ang I $(11,12)$. A penultimate proline in peptides prevents ACE from cleaving a dipeptide from the $\mathrm{COOH}$ terminus [Pro ${ }^{11} \mathrm{DAla}{ }^{12}$ ] Ang I and a carboxy-terminal DAla was added to prevent carboxypeptidases from making the penultimate proline into a $\mathrm{COOH}$-terminal proline. Introducing Pro-DAla to the $\mathrm{COOH}$ terminus of Ang I also reduced the intrinsic activity of this Ang I analog for the Ang II receptor by a 100-fold compared to that of Ang I (12). In in vitro biochemical studies, chymase, but not ACE, converted [Pro ${ }^{11}$ DAla ${ }^{12}$ ] Ang I to Ang II. Using isolated human cardiac trabeculae, we have shown that $\left[\right.$ Pro $^{11} \mathrm{DAla}{ }^{12}$ ] Ang I produces a positive inotropic response which can be inhibited by Ang II receptor blockade (12). Finally, in addition to the use of a chymase selective substrate, the use of a conscious baboon model for demonstrating chymase dependent Ang II formation circumvents potential problems related to the premature release of chymase from mast cells due to anesthesia or tissue handling. These studies provide evidence that chymase is functional in tissues of conscious baboons and represent an important step in understanding the role of the chymase-angiotensin system in primates.

\section{Methods}

\section{Animal instrumentation}

Nine adult baboons ( 7 males, 2 females), weighing to $16-27 \mathrm{~kg}$ were pre-instrumented for physiologic monitoring in the conscious state using methods previously described (13). Briefly, after sedation with ketamine $(10 \mathrm{mg} / \mathrm{kg})$ and atropine $(0.5 \mathrm{mg})$, the animals were intubated and anesthesia maintained with $1.0-1.5 \%$ halothane. A Konigsburg micromanometer (P5-P7) and a polyvinyl catheter (O.D .095 mm, I.D $.066 \mathrm{~mm}$ ) were implanted in the left ventricular (LV) apex and miniaturized sonomicrometer pairs were placed in the endocardium across the $\mathrm{LV}$ anteroposterior minor axis ( $3 \mathrm{MHz}, 6 \mathrm{~mm}$ ) and transmurally at the mid LV free wall for measurement of wall thickness ( 5 MHz, $2.5 \mathrm{~mm}$ ). A polyvinyl catheter (O.D. $.095 \mathrm{~mm}$, I.D. $.066 \mathrm{~mm}$ ) was implanted in the right atrial appendage for central venous access. Wires and tubes were tunnelled subcutaneously into the interscapular area for later attachment to a tether system. Postoperative pain was reduced by the use of Buprenet $(0.01 \mathrm{mg} / \mathrm{kg}$ I.M., q. $6 \mathrm{~h})$ and postoperative antibiotics (Monocid $25 \mathrm{mg} / \mathrm{kg}$ ) were administered for $5 \mathrm{~d}$ to reduce the risk of infection. After a minimum of $1 \mathrm{wk}$, studies were performed with the animal resting quietly in an individual tether cage. Wires were run from the tether cage into an adjoining room equipped with an eight channel physiologic recorder and a microprocessor for A-D conversion of pressure and dimension signals.

\section{Hemodynamic data acquisition and analysis}

The micromanometer and fluid-filled catheters were calibrated before implantation with a mercury manometer. Zero drift of the micromanometer was corrected by matching the $\mathrm{LV}$ end diastolic pressure measured simultaneously through the LV catheter. The fluid-filled LV catheter was connected to a pre-calibrated Statham $23 \mathrm{~dB}$ transducer (housed in the connector box of the tether jacket) with zero pressure at the level of the mid right atrium. The transit time of ultrasound between the ultrasonic dimension crystals was measured with a multichannel sonomicrometer (Triton Technology, Inc.) and converted to distance assuming a constant velocity of sound and blood of $1.55 \mathrm{~mm} / \mu \mathrm{sec}$.

Analog signals for high fidelity and fluid-filled LV pressures, LV short axis and transmural dimensions, $\mathrm{LV} \mathrm{dP} / \mathrm{dt}$ and the electrocardiogram were recorded on-line on a Gould multichannel recorder (Gould, Cleveland, $\mathrm{OH}$ ) and digitized through an A-D board (Dual Control Systems) interfaced to an IBM AT computer at $500 \mathrm{~Hz}$ and stored on floppy disc. Data were analyzed using algorithms and software developed in our laboratory (14).

Fractional shortening of the LV minor axis was calculated as (EDD$\mathrm{ESD}) / \mathrm{EDD}$, where $\mathrm{EDD}=\mathrm{LV}$ end diastolic dimension and $\mathrm{ESD}=\mathrm{LV}$ end systolic dimension. Fractional LV wall thickening was calculated as

\section{$($ ESWTh - EDWTh)/ESWTh}

where ESWTh $=$ LV end systolic wall thickness and EDWTh $=$ LV end diastolic wall thickness.

Steady state data were acquired over $10 \mathrm{~s}$ and averaged.

The analog LV dP/dt signal was obtained by electronic differentiation of the high fidelity LV pressure signal. Left ventricular end diastole was defined as the time that $+\mathrm{LV} \mathrm{dP} / \mathrm{dt}$ increased by at least 150 $\mathrm{mmHg} / \mathrm{s}$ for $50 \mathrm{~ms}$, and $\mathrm{LV}$ end systole was defined as the time of the maximum ratio of pressure to dimension. The time constant of LV relaxation was derived from the high fidelity LV pressure tracing using the method of Weiss et al. (15), which assumes a non-zero asymptote and has been shown to be directionally equivalent to other mathematical approaches for quantitation of isovolumic pressure decay (16).

[Pro ${ }^{11}$ DAla $\left.{ }^{12}\right]$ Ang I synthesis and purification

[Pro ${ }^{11} \mathrm{DAla}^{12}$ ] Ang I used in this study was synthesized by Dr. K. S. Misono, Cleveland Clinic Foundation. This peptide was purified on a C18 reverse-phase HPLC column $(2.2 \times 25 \mathrm{~cm}$; Vydac $)$ with a linear acetonitrile gradient containing $0.1 \%$ trifluroacetic acid and characterized by amino acid analysis and by analytical C18 reverse-phase HPLC $\left[\right.$ Pro $^{11}{ }^{1} \mathrm{DAla}^{12}$ ] Ang I was purified to a peptide purity exceeding $99 \%$.

\section{Experimental protocols}

Hemodynamic effects of $\left[\right.$ Pro $\left.^{11} \mathrm{DAla}^{12}\right]$ Ang I infusion. Animals were allowed to acclimate to the tether system for at least $2 \mathrm{~d}$. Studies were performed with the animal resting quietly in an individual tether cage After baseline hemodynamic data were acquired, animals were given the chymase-specific substrate, [Pro ${ }^{11}$ DAla ${ }^{12}$ ] Ang I sequentially as a $0.1 \mathrm{mg}$ intravenous bolus, an hour long infusion of $5 \mathrm{mg}$ (in $75 \mathrm{cc}$ D5W) and as a $3 \mathrm{mg}$ bolus (Group $\mathrm{A}, n=9$ ). In order to determine whether the hemodynamic effects were Ang II-mediated, the specific Ang II-receptor antagonist, losartan (Merck-Dupont) was given within three minutes of the $3 \mathrm{mg}$ bolus of [Pro ${ }^{11} \mathrm{DAla}{ }^{12}$ ] Ang I. In a subgroup of animals (Group $\mathrm{B}, \mathrm{N}=6$ ), the same protocol was repeated after ACE blockade one to $2 \mathrm{~d}$ later. Captopril was given as a $5 \mathrm{mg}$ i.v. bolus, followed in $10 \mathrm{~min}$ by $15 \mathrm{mg}$ over $1 \mathrm{~h}$, administered as a coinfusion with [Pro ${ }^{11} \mathrm{DAla}^{12}$ ] Ang I. This dose of captopril was derived empirically in pilot studies and was associated with measurable hemodynamic effects (see Table II).

\section{Myocardial effects of [Pro ${ }^{11}$ DAla $\left.{ }^{I 2}\right]$ Ang I and Ang II}

To determine the direct myocardial effects of locally generated Ang II, four animals underwent selective left coronary artery catheterization under light general anesthesia (ketamine $10 \mathrm{mg} / \mathrm{kg} / \mathrm{I} . \mathrm{M}$. and halothane $0.5-1 \%$ ). Incremental doses (5 to $100 \mu \mathrm{g}$ ) of [Pro $\left.{ }^{11} D A a^{12}\right]$ Ang I were suspended in $1 \mathrm{cc}$ saline, mixed with $1 \mathrm{cc}$ radiographic contrast (16) and given directly through the coronary catheter. Intracoronary Ang II $(0.7$ and $1.4 \mu \mathrm{g})$ was used as a positive control in an additional four normotensive instrumented animals. 

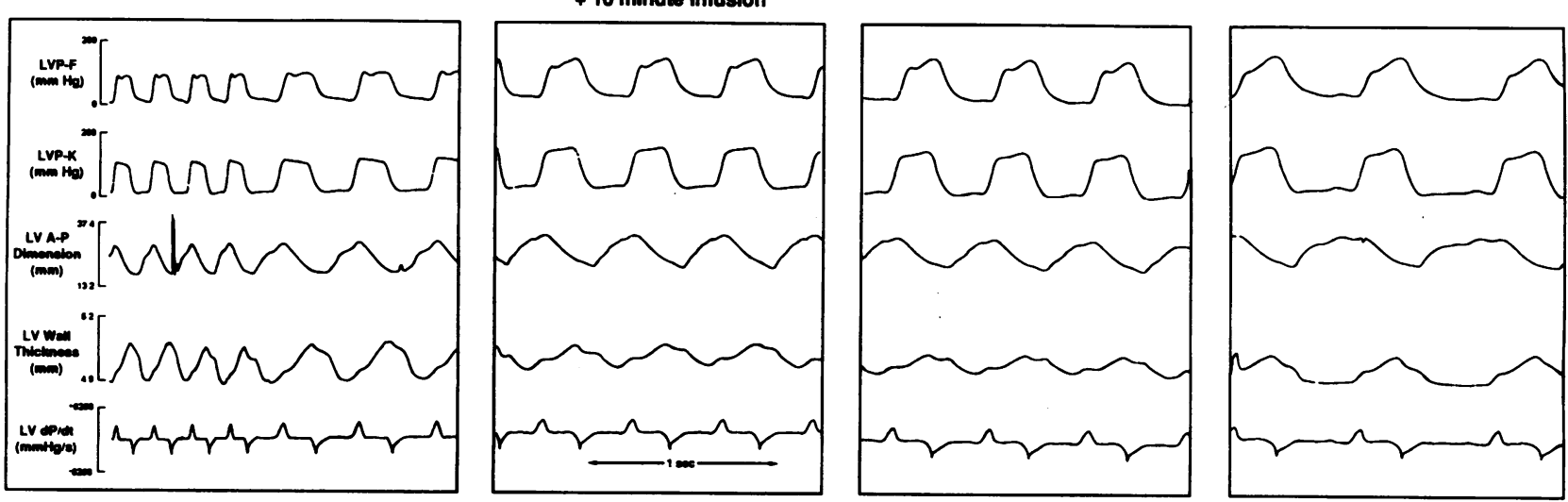

Figure 1. Representative analog recordings during baseline, after a $0.1 \mathrm{mg}$ bolus, constant infusion, and $3 \mathrm{mg}$ bolus of [Pro ${ }^{11} \mathrm{DAla}{ }^{12}$ ] Ang I. Note the increase in LV systolic and diastolic pressures and dimensions and the decreased LV fractional shortening and wall thickening. Despite the increased LV end diastolic pressure, there is no change in LV peak (+) dP/dt. PDA, [Pro ${ }^{11} D A{ }^{12}{ }^{12}$ ] Ang I; $L V P-F, L V$ pressure from fluid-filled catheter; $L V P-K, L V$ pressure from high fidelity Konigsburg catheter; $A-P$, anteroposterior minor axis; $d P / d t$, time derivative of $L V$ pressure.

Enzymatic assay for chymase-like activity in baboon tissues

At necropsy, samples from heart, lung, aorta, femoral artery, skeletal muscle, GI tract, kidney, adrenal and brain were harvested to determine the tissue distribution of the chymase. The extraction of chymase from each tissue was performed as follows (8): a half gram of each tissue

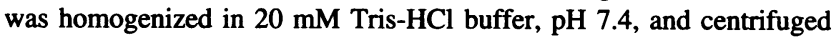
at $40,000 \mathrm{~g}$ for $20 \mathrm{~min}$. This procedure was repeated twice. The pellet was resuspended in $2 \mathrm{ml}$ of $20 \mathrm{mM}$ Tris- $\mathrm{HCl}$ buffer, $\mathrm{pH} \mathrm{8.0,} \mathrm{containing}$ $2.0 \mathrm{M} \mathrm{KCl}$ and $1 \%$ Triton X-100. $10 \mu \mathrm{l}$ samples, preincubated for 30 min at room temperature with $1 \mathrm{mM}$ EDTA, $1 \mathrm{mM} o$-phenanthroline, $10 \mu \mathrm{M}$ aprotinin, and with or without $100 \mu \mathrm{M}$ chymostatin, were incubated for $20 \mathrm{~min}$ at $37^{\circ} \mathrm{C}$ with $20 \mathrm{nmol}$ [Pro ${ }^{11} \mathrm{DAla}^{12}$ ] Ang I in $50 \mu \mathrm{l}$ of $20 \mu \mathrm{M}$ Tris- $\mathrm{HCl}$ buffer, $\mathrm{pH} 8.0$, containing $0.5 \mathrm{M} \mathrm{KCl}$ and $0.01 \%$ Triton X-100. Generated Ang II was analyzed using a C18 reverse phase HPLC column (Vydac). The peak area corresponding to a synthetic Ang II standard was integrated to calculate Ang II formation. Chymostatininhibitable Ang II formation was considered to represent the chymaselike activity and was expressed as pmol of Ang II formed $/ \mathrm{min} / \mathrm{g}$ tissue wet weight. Other known Ang II forming enzymes including ACE, cathespin G, kallikrein, chymotrypsin, trypsin, and carboxypeptidases are completely inhibited in this assay procedure.

\section{Statistics}

In each group, hemodynamic and dimension data were compared using a one-way repeated measures analysis of variance (StatView, Abacus Concepts, Berkeley, CA). When significant differences were found, group means were compared with Scheffes $F$ test. The effects of ACE blockade and [Pro ${ }^{11} \mathrm{DAla}{ }^{12}$ ] Ang I on the percent change from baseline for each hemodynamic and dimension variable was examined in Group $B$ animals with two factor repeated measures ANOVA (SuperAnova, Abacus Concepts). A $P$ value of $<0.05$ was considered significant. Data are expressed as mean \pm SD.

\section{Results}

Hemodynamic effects of [Pro ${ }^{11} D A$ la $\left.^{12}\right]$ Ang I. Analog recordings from a representative experiment are shown in Fig. 1 and the results from nine baboons (Group A) are summarized in Table I. Intravenous administration of [Pro ${ }^{11} \mathrm{DAla}^{12}$ ] Ang I significantly increased LV systolic and diastolic pressures, LV end-diastolic and end-systolic dimensions and the time constant of isovolumic LV relaxation, and significantly decreased LV fractional shortening and wall thickening. Despite increases in

Table I. Systemic Effects [Pro ${ }^{11}$ DAla ${ }^{12}$ ] Ang I on LV Performance in Conscious Baboons $(n=9)$

\begin{tabular}{|c|c|c|c|c|c|}
\hline & Baseline & $\begin{array}{l}0.1 \mathrm{mg} \\
\text { Bolus }\end{array}$ & $\begin{array}{c}\text { Maximum } \\
\text { infusion }\end{array}$ & $\begin{array}{l}3 \mathrm{mg} \\
\text { Bolus }\end{array}$ & $\begin{array}{l}\text { Ang II receptor } \\
\text { antagonist }\end{array}$ \\
\hline LV systolic $\mathrm{P}$ (mmHg) & $118.8 \pm 14.0$ & $124.6 \pm 12.9$ & $151.8 \pm 21.9^{* \pm \|}$ & $191.2 \pm 40.4^{* \neq \S \|}$ & $117.6 \pm 14.2$ \\
\hline LV diastolic $\mathrm{P}(\mathrm{mmHg})$ & $9.3 \pm 3.4$ & $12.6 \pm 4.7$ & $20.0 \pm 3.5 * \neq 8$ & $29.2 \pm 5.6 * \pm \S \|$ & $9.3 \pm 4.0$ \\
\hline $\mathrm{LV} \mathrm{dP/dt} \mathrm{(mmHg/s)}$ & $2445 \pm 384$ & $2471 \pm 438$ & $2387 \pm 398$ & $2724 \pm 776$ & $2549 \pm 610$ \\
\hline Tau (ms) & $24.6 \pm 6.5$ & $26.7 \pm 5.5$ & $30.9 \pm 5.9$ & $41.9 \pm 11.6^{* \neq 8 \|}$ & $27.9 \pm 8.1$ \\
\hline Heart rate $(\mathrm{bpm})$ & $115 \pm 19$ & $123 \pm 29$ & $113 \pm 19$ & $115 \pm 34$ & $125 \pm 32$ \\
\hline LVEDD (mm) & $26.9 \pm 7.8$ & $27.5 \pm 7.9$ & $28.9 \pm 8.4^{* \pm}$ & $29.2 \pm 8.3^{* \neq \S} \|$ & $26.7 \pm 7.8$ \\
\hline $\operatorname{LVESD}(\mathrm{mm})$ & $19.9 \pm 7.2$ & $20.9 \pm 7.0$ & $22.5 \pm 7.6 * \pm \|$ & $23.5 \pm 7.9 * \pm 8 \|$ & $20.6 \pm 7.7$ \\
\hline Fractional LV shortening (\%) & $28.1 \pm 8.9$ & $25.3 \pm 6.5$ & $23.4 \pm 7.4 *$ & $20.3 \pm 7.3 * \pm 8 \|$ & $24.6 \pm 7.3$ \\
\hline Fractional LV wall thickening (\%) & $22.4 \pm 7.8$ & $20.4 \pm 6.9$ & $17.9 \pm 6.6$ & $15.4 \pm 4.9 * 8$ & $21.7 \pm 8.8$ \\
\hline
\end{tabular}

$L V$, left ventricular; $P$, pressure; tau, time constant of isovolumic LV relaxation; $E D D$, end diastolic dimension; $E S D$, end systolic dimension; Ang II antagonist, losartan $(1 \mathrm{mg} / \mathrm{kg}) .{ }^{*} P<0.05$ vs. baseline; ${ }^{\ddagger}$ vs. $0.1 \mathrm{mg}$ bolus; ${ }^{8} \mathrm{vs.} \mathrm{max;} \mathrm{"} \mathrm{vs.} \mathrm{inhibitor.}$ 
Table II. Systemic Effects of Intravenous [Pro $\left.{ }^{11} D A a^{12}\right]$ Ang I on LV Performance after ACE Inhibition in Conscious Baboons ( $n=6$ )

\begin{tabular}{|c|c|c|c|c|c|c|}
\hline & \multicolumn{2}{|c|}{ Baseline } & \multirow[b]{2}{*}{$\begin{array}{c}0.1 \mathrm{mg} \\
\text { Bolus }\end{array}$} & \multirow[b]{2}{*}{$\begin{array}{c}\text { Maximum } \\
\text { infusion }\end{array}$} & \multirow[b]{2}{*}{$\begin{array}{l}3 \mathrm{mg} \\
\text { Bolus }\end{array}$} & \multirow[b]{2}{*}{$\begin{array}{l}\text { Ang II receptor } \\
\text { antagonist }\end{array}$} \\
\hline & Pre captopril & Post captopril & & & & \\
\hline LV systolic $\mathrm{P}(\mathrm{mmHg})$ & $115.8 \pm 17.6$ & $109.1 \pm 18.7^{1}$ & $112.8 \pm 21.6$ & $147.3 \pm 29.1 * \pm \S$ & $197.7 \pm 57.6^{* \pm \S} \|$ & $117.8 \pm 11.4$ \\
\hline LV diastolic $\mathrm{P}(\mathrm{mmHg})$ & $10.3 \pm 2.5$ & $9.6 \pm 2.1$ & $9.5 \pm 1.1$ & $17.2 \pm 3.0^{* \pm 8}$ & $29.5 \pm 5.2^{* \pm \S \|}$ & $10.5 \pm 3.7$ \\
\hline $\mathrm{LV} \mathrm{dP} / \mathrm{dt}(\mathrm{mmHg} / \mathrm{s})$ & $2118.2 \pm 283.7$ & $2176 \pm 349$ & $2083 \pm 264$ & $2041 \pm 329$ & $2795 \pm 1019$ & $2210 \pm 341$ \\
\hline Tau (ms) & $25.84 \pm 3.57$ & $29.1 \pm 6.9$ & $28.6 \pm 4.7$ & $35.6 \pm 5.0$ & $44.0 \pm 10.3^{* \neq \S}$ & $29.4 \pm 8.5$ \\
\hline Heart rate (bpm) & $102.8 \pm 15.6$ & $116 \pm 11$ & $112 \pm 16$ & $105 \pm 12$ & $96 \pm 25$ & $112 \pm 18$ \\
\hline LVEDD (mm) & $29.3 \pm 6.6$ & $29.6 \pm 7.1$ & $29.7 \pm 7.1$ & $31.4 \pm 6.9 * \neq 8$ & $32.3 \pm 7.2^{* \neq \S}$ & $29.3 \pm 6.4$ \\
\hline $\operatorname{LVESD}(\mathrm{mm})$ & $22.9 \pm 6.7$ & $22.6 \pm 7.3$ & $23.2 \pm 7.2$ & $25.3 \pm 7.0^{* \neq \S}$ & $26.1 \pm 6.7^{* \neq \S}$ & $23.0 \pm 6.8$ \\
\hline Fractional LV shortening (\%) & $22.9 \pm 6.1$ & $24.8 \pm 6.4^{9}$ & $23.0 \pm 6.2$ & $20.3 \pm 5.8^{*}$ & $19.3 \pm 5.0^{*}$ & $22.6 \pm 6.0$ \\
\hline Fractional LV wall thickening (\%) & $21.23 \pm 9.6$ & $22.0 \pm 9.6^{9}$ & $21.0 \pm 10.6$ & $18.3 \pm 10.9$ & $15.0 \pm 5.7$ & $21.0 \pm 10.8$ \\
\hline
\end{tabular}

${ }^{*} P<0.05$ vs. baseline; ${ }^{\ddagger}$ vs. $0.1 \mathrm{mg}$ bolus; ${ }^{\S}$ vs. max; " vs. inhibitor; " pre vs. post captopril (5 mg I.V.) Abbreviations as in Table I.

$\mathrm{LV}$ end diastolic pressure and dimension, $\mathrm{LV} \mathrm{dP} / \mathrm{dt}$ was unchanged. Heart rate was unchanged by [Pro ${ }^{11}$ DAla ${ }^{12}$ ] Ang I administration, despite the elevation of LV systolic pressure and the expected baroreceptor-mediated cardiac slowing.

Maximum hemodynamic effects during the hourlong infusion of [Pro $\left.{ }^{11} \mathrm{DAla}{ }^{12}\right]$ Ang I occurred after 50 $\pm 7 \mathrm{~min}$. After an intravenous bolus, hemodynamic and LV dimension changes generally occurred within $15 \mathrm{~s}$.

All hemodynamic variables returned to baseline levels immediately after administration of losartan (Fig. 2, Table I), indicating that the hemodynamic effects of [Pro $\left.{ }^{11} \mathrm{DAla}^{12}\right]$ Ang I were dependent on Ang II production.

Influence of ACE blockade. Pretreatment with intravenous captopril decreased significantly LV systolic pressure, fractional shortening and wall thickening (Table II). Heart rate tended to increase while $\mathrm{LV} \mathrm{dP/dt}$ and the time constant of $\mathrm{LV}$ relaxation were unchanged. The hemodynamic effects of intravenous [Pro${ }^{11} \mathrm{DAla}^{12}$ ] Ang I in the six baboons studied during ACE blockade (Group B) are summarized in Table II and hemodynamic and dimension variables from paired experiments with and without ACE blockade are compared as the percent change from baseline values in Table III and Fig. 3. The ACE inhibitor captopril failed to attenuate any of the hemodynamic and LV functional effects of [Pro ${ }^{11}$ DAla $\left.{ }^{12}\right]$ Ang I.

Direct myocardial effects of [Pro ${ }^{11}$ DAla $\left.{ }^{12}\right]$ Ang I and Ang II. An analog recording from a selective left intracoronary injection of [Pro ${ }^{11} \mathrm{DAla}^{12}$ ] Ang $\mathrm{I}$ is shown in Fig. 4 and data from four baboons are summarized in Table IV. In doses from 5 to $40 \mu \mathrm{g}$, [Pro ${ }^{11} \mathrm{DAla}^{12}$ ] Ang I did not have a consistent effect on systemic hemodynamics or LV function. At doses of $100 \mu \mathrm{g}$,
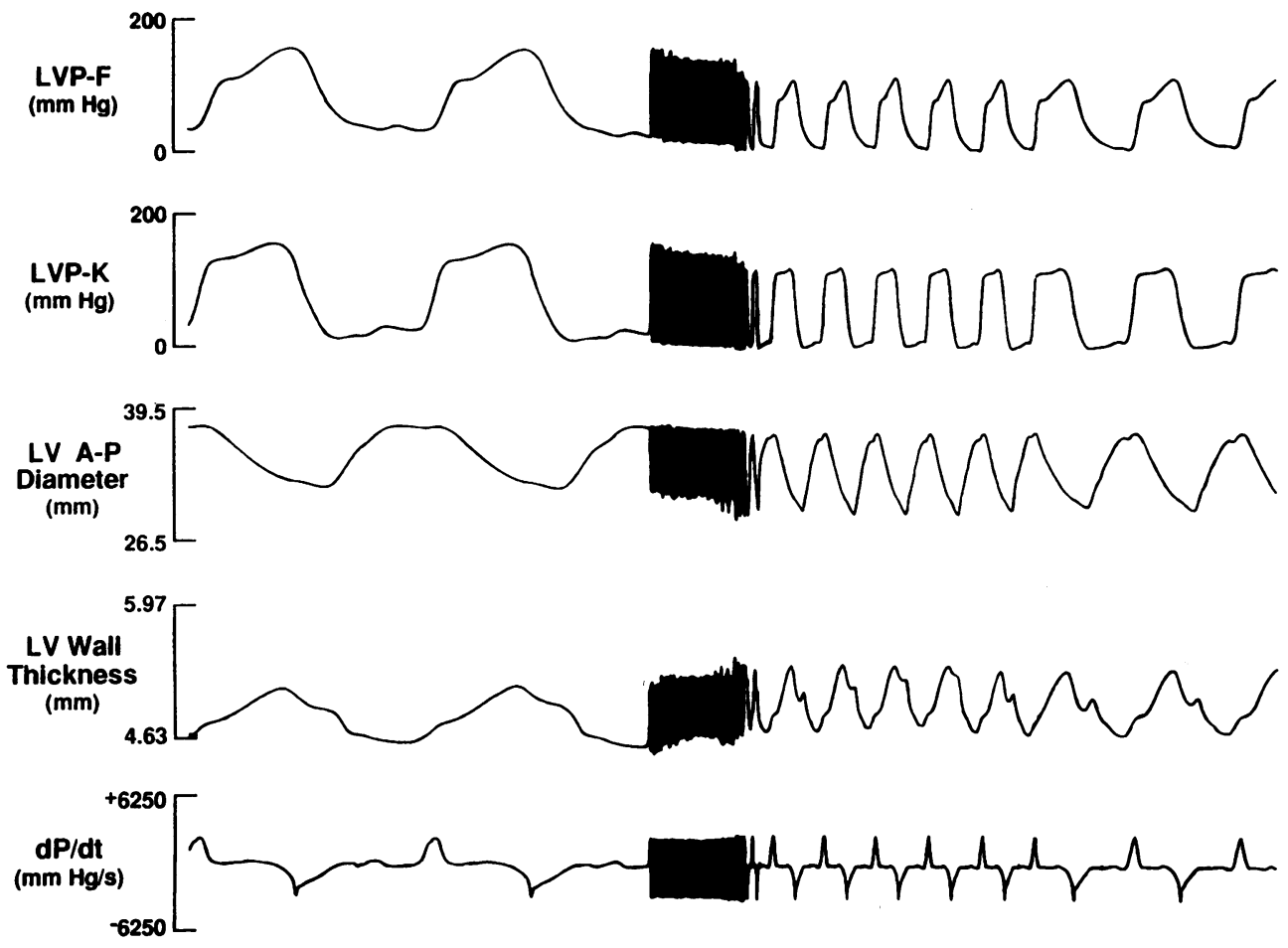

Figure 2. Analog recording immediately before and after administration of the Ang II receptor antagonist, losartan. Note the prompt normalization of hemodynamics and left ventricular mechanics. Abbreviations as in Fig. 1. 
Table III. Percent Change of Hemodynamic and Dimension Variables from Baseline: Influence of ACE Blockade Group B ( $n=6)$

\begin{tabular}{|c|c|c|c|c|c|c|c|c|}
\hline & \multicolumn{2}{|c|}{$0.1 \mathrm{mg}$ bolus } & \multicolumn{2}{|c|}{ Maximum infusion } & \multicolumn{2}{|c|}{$3 \mathrm{mg}$ bolus } & \multicolumn{2}{|c|}{$\begin{array}{l}\text { Ang II receptor } \\
\text { antagonist }\end{array}$} \\
\hline & Cap - & Cap + & Cap - & Cap + & Cap - & Cap + & Cap - & Cap + \\
\hline LV systolic $P$ & $6 \pm 4$ & $3 \pm 7$ & $36 \pm 19$ & $35 \pm 11$ & $74 \pm 37$ & $78 \pm 26$ & $7 \pm 15$ & $10 \pm 13$ \\
\hline LV diastolic P & $6 \pm 18$ & $4 \pm 26$ & $100 \pm 43$ & $88 \pm 63$ & $227 \pm 61$ & $221 \pm 96$ & $-2 \pm 35$ & $17 \pm 57$ \\
\hline $\mathrm{LV} \mathrm{dP} / \mathrm{dt}$ & $2 \pm 6$ & $-4 \pm 4$ & $-3 \pm 13$ & $-6 \pm 7$ & $32 \pm 46$ & $28 \pm 40$ & $-4 \pm 6$ & $3 \pm 17$ \\
\hline Tau & $2 \pm 14$ & $0 \pm 11$ & $28 \pm 23$ & $24 \pm 12$ & $61 \pm 25$ & $53 \pm 27$ & $7 \pm 16$ & $2 \pm 18$ \\
\hline Heart rate & $4 \pm 16$ & $-4 \pm 10$ & $-5 \pm 10$ & $-9 \pm 8$ & $-1 \pm 27$ & $-18 \pm 18$ & $0 \pm 20$ & $-3 \pm 16$ \\
\hline LVEDD & $2 \pm 3$ & $0 \pm 2$ & $9 \pm 5$ & $7 \pm 4$ & $10 \pm 6$ & $9 \pm 4$ & $4 \pm 7$ & $-1 \pm 4$ \\
\hline LVESD & $2 \pm 2$ & $3 \pm 1$ & $12 \pm 5$ & $13 \pm 8$ & $15 \pm 6$ & $18 \pm 8$ & $4 \pm 4$ & $2 \pm 3$ \\
\hline Fractional LV shortening & $-3 \pm 4$ & $-9 \pm 7$ & $-13 \pm 9$ & $-16 \pm 11$ & $-22 \pm 25$ & $-24 \pm 9$ & $-9 \pm 16$ & $5 \pm 19$ \\
\hline Fractional LV wall thickening & $-2 \pm 15$ & $-6 \pm 12$ & $-13 \pm 22$ & $-19 \pm 18$ & $-20 \pm 30$ & $-29 \pm 14$ & $-7 \pm 17$ & $-7 \pm 17$ \\
\hline
\end{tabular}

$A C E$, angiotensin converting enzyme; Cap, captopril; other abbreviations as in Table I.

intracoronary injection caused delayed (30-60 s) systemic vascular effects, although delayed effects were occasionally seen at lower doses.

Results from the intracoronary Ang II experiments are summarized in Fig. 5. Ang II produced dose-dependent delayed systemic effects similar to those observed with [Pro ${ }^{11} \mathrm{DAla}^{12}$ ] Ang I-i.e., increases in LV systolic and diastolic pressure and the time constant of LV relaxation, decreases in LV fractional shortening and no changes in either heart rate or peak positive LV dP/dt. Direct positive inotropic effects (i.e., increases in $\mathrm{LV}[+] \mathrm{dP} / \mathrm{dt}$ and shortening) were not observed.

Tissue distribution of chymase-like activity. A summary of chymase-like activity in several tissues from normal and renal artery-clipped baboons is presented in Table V. Additional baboon tissues were made available from an unrelated study. Chymase-like activity was widely distributed in normal and hypertensive baboons. The highest levels of chymase-like activity were observed in the spleen and lungs; most other tissues contained levels of chymase-like activity between 0.05 and $0.56 \%$ of that found in the spleen. Levels of chymase-like activity in cardiac ventricles and atria were intermediate $(\sim 30 \mathrm{pmol} / \mathrm{min} /$ g tissue wet weight). Compared with normotensive baboons, chymase-like activity from hypertensive baboons was significantly less in the aorta and greater in the spleen. Levels of chymase-like activity in cardiac ventricles and atria (and other tissues in which a sufficient number of samples permitted comparisons) were similar (Table V).

\section{Discussion}

In this study, we provide the first in vivo evidence of an ACEindependent pathway for Ang II production. Infusion of the Ang I analog, [Pro ${ }^{11}$ DAla ${ }^{12}$ ] Ang I, a selective and specific substrate for human chymase but not ACE, produced hemodynamic and left ventricular functional changes consistent with systemic arterial vasoconstriction; the latter is an action of Ang II which is highly conserved in all mammals (17). Although we demonstrated identical hemodynamic responses with [Pro ${ }^{11-}$ DAla $^{12}$ ] Ang I infusion after pretreatment with captopril, we cannot be certain that plasma ACE was suppressed entirely or that tissue ACE was blocked. However, the absence of any attenuation of the hemodynamic changes by ACE inhibition

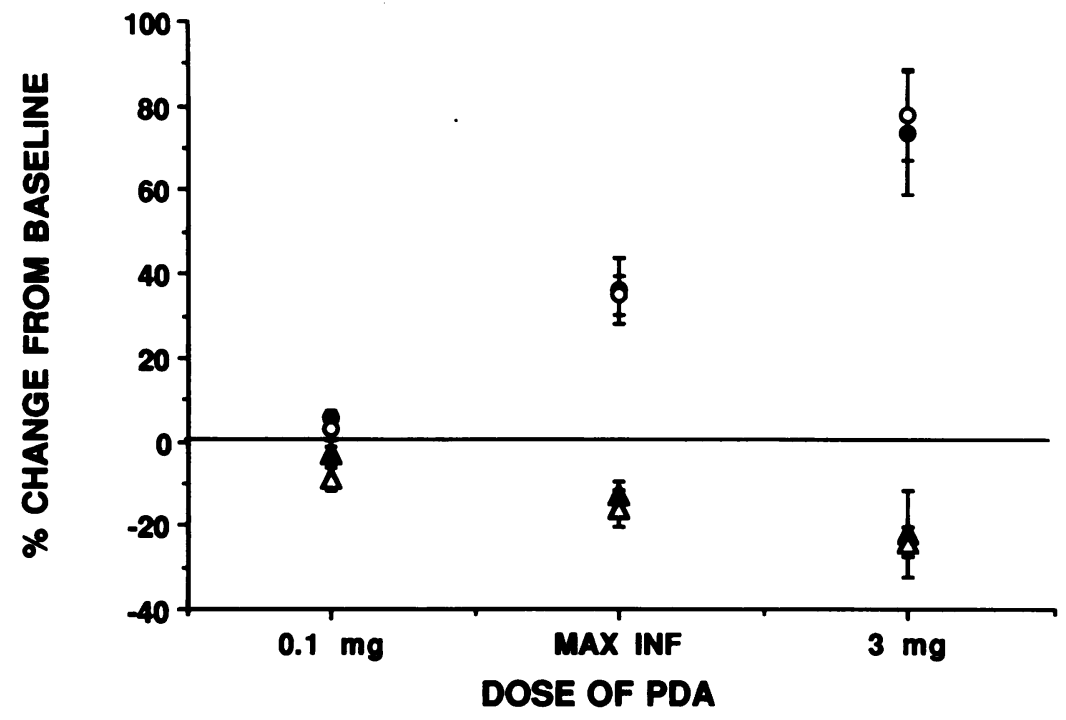

Figure 3. Changes in LV systolic pressure and fractional shortening in response to graded doses of [ Pro $^{11} \mathrm{DAla}^{12}$ ] Ang I (PDA) before (closed circles and triangles, respectively) and after (open circles and triangles, respectively) ACE inhibition, expressed as a percent change from baseline values. 


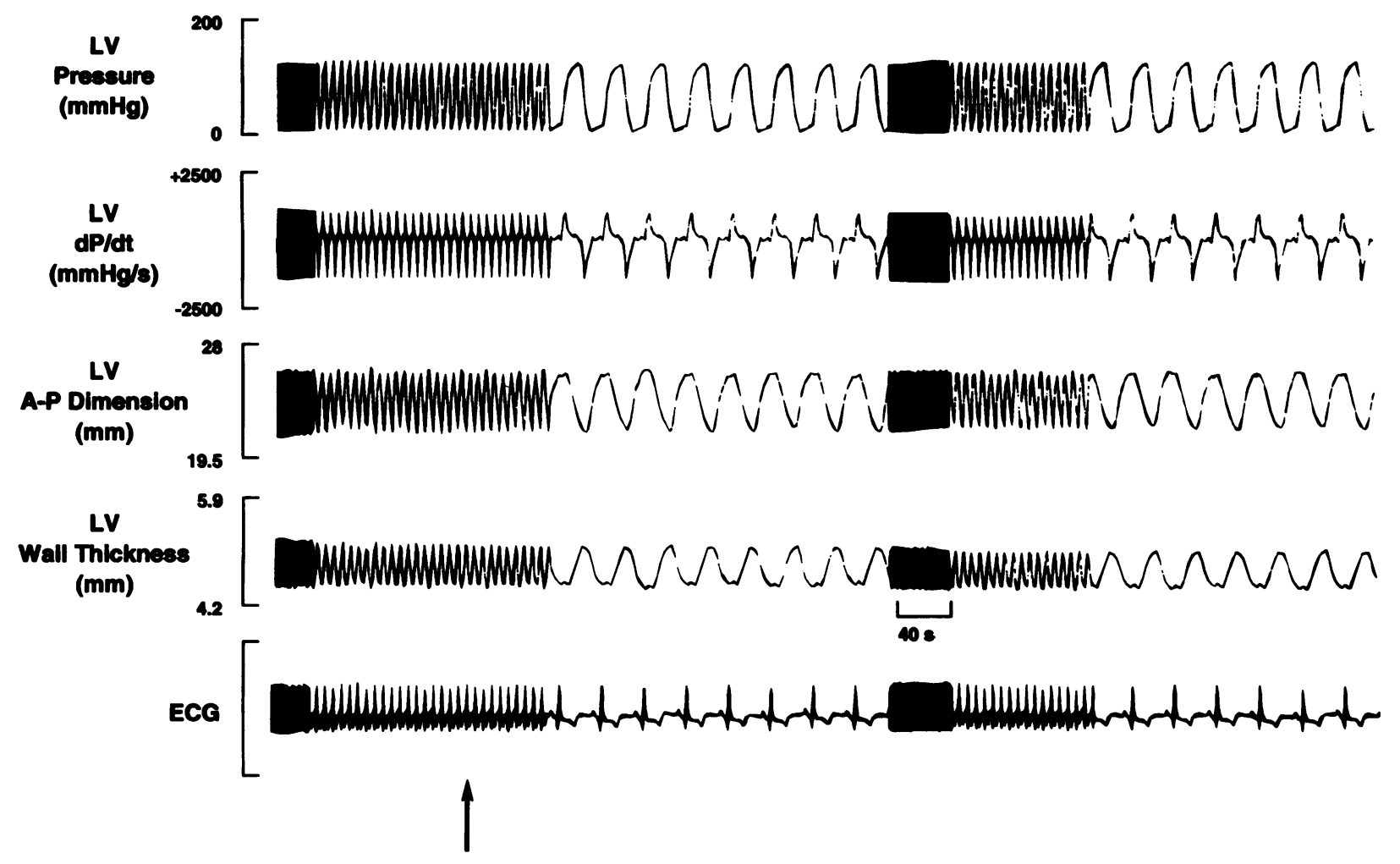

Figure 4. Analog recording demonstrating the effects of an $80 \mu \mathrm{g}$ intracoronary injection of [Pro "DAla ${ }^{12}$ ] Ang I (arrow). Note the delayed increase in LV pressures and dimensions and decreased LV fractional shortening and wall thickening. ECG, electrocardiogram. Other abbreviations as in Fig. 1.

suggests that Ang II was generated at least in part, by an ACEindependent pathway. Thus, our data indicate that the hemodynamic effects we observed were the result of functional chymase-like activity in baboon tissues. This contention is supported by our additional finding in the postmortem studies; i.e., that chymase-like activity is present in several baboon tissues, including the heart and the aorta. To ensure that the hemodynamic effects we observed were not mediated by vasoactive peptides other than Ang II, the specific nonpeptide Ang II inhibitor, losartan, was given after a large bolus of [Pro ${ }^{11} \mathrm{DAla}^{12}$ ]
Ang I. This caused a prompt and complete reversal of the hemodynamic and LV functional abnormalities. Moreover, [Pro ${ }^{11-}$ DAla $^{12}$ ] Ang I does not interact directly with the Ang II receptor $($ IC50 $>10 \mathrm{pM})(11)$. Taken together, these data indicate that [Pro ${ }^{11}$ DAla ${ }^{12}$ ] Ang I-induced hemodynamic changes were caused by ACE-independent production of Ang II. Because of the unique substrate specificity. of human heart chymase (15) and the abundance of chymase-like activity in baboon cardiovascular tissues, we speculate that chymase is likely responsible for Ang II production in our study. However, because of the

Table IV. Effects of Graded Intracoronary Doses of $\left[\right.$ Pro $\left.^{I I} D A l a^{12}\right]$ Ang I on LV Performance in Anesthetized Baboons $(n=4)$

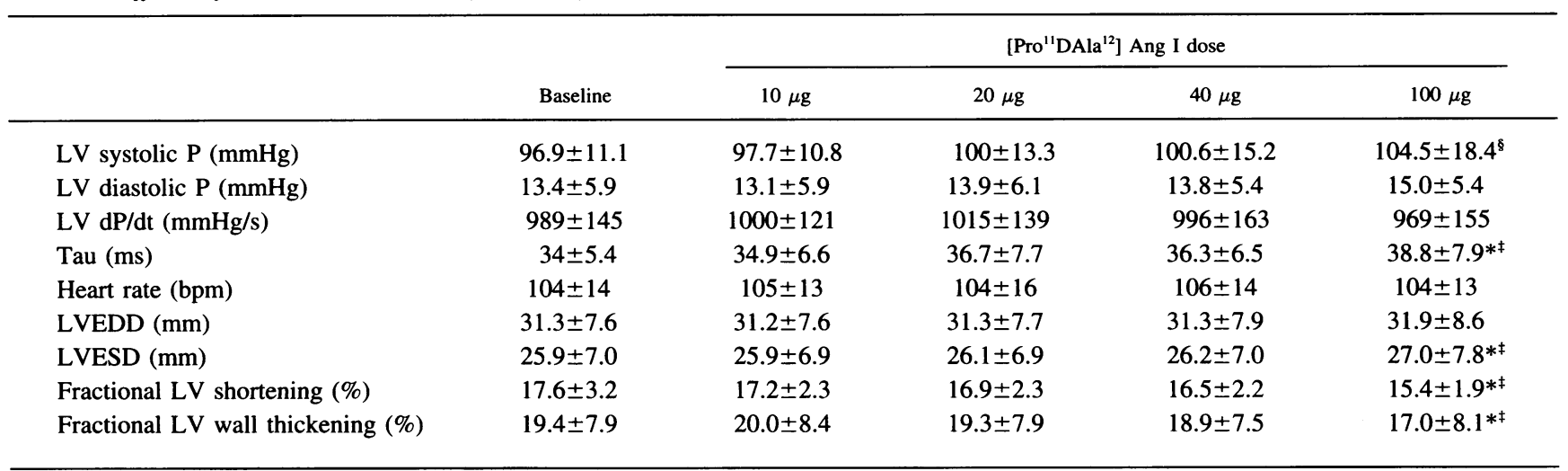

${ }^{*} P<0.05$ vs. baseline; ${ }^{*} P<0.05$ vs. $10 \mu \mathrm{g} ;{ }^{\S} P=.06$ vs. baseline. Abbreviations as in Table $\mathrm{I}$. 


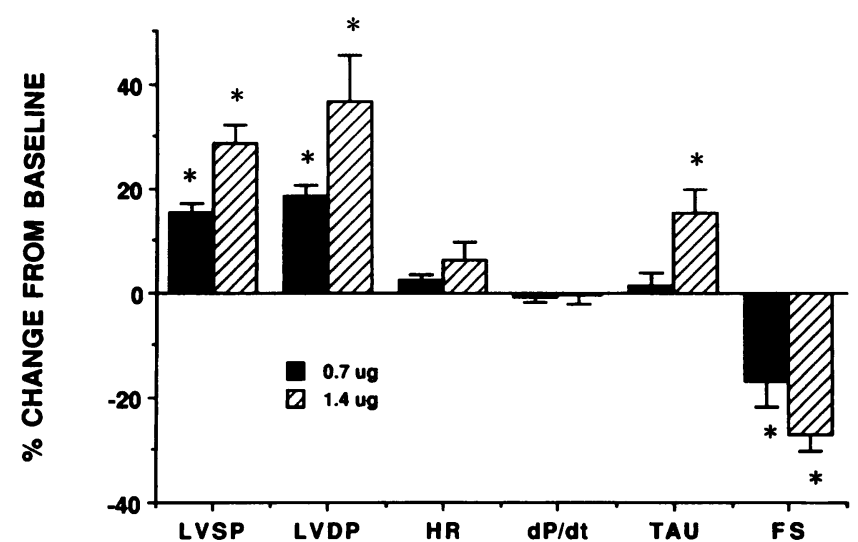

Figure 5. Changes in LV systolic (LVSP) and diastolic (LVDP) pressures, heart rate $(H R), L V$ peak $(+) \mathrm{dP} / \mathrm{dt}$, the time constant of $L V$ relaxation (Tau) and LV fractional shortening (FS) in response to 0.7 and $1.4 \mu \mathrm{g}$ intracoronary boluses of Ang II. Data are expressed as the percent change from baseline values. ${ }^{*} P<0.05$ vs. no change from baseline.

unavailability of a specific chymase inhibitor, we cannot explicitly rule out the contribution of other enzymes in the [Pro ${ }^{11-}$ $\mathrm{DAla}^{12}$ ] Ang I response.

In addition to its potent vasoconstrictor effect, Ang II modulates directly and indirectly cardiac rate, contractility and myocyte hypertrophy and proliferation. Ang II has a direct positive inotropic effect in some, but not all, isolated heart preparations (18-24) in which indirect Ang II sympathetic facilitation was excluded. For example, a positive inotropic effect has been demonstrated in isolated hearts from dogs $(19,20)$, cats $(21)$, rabbits (22), chickens (22), and humans (23), but not in guinea pigs or adult rats (24). In contrast, a positive inotropic effect has been difficult to demonstrate in the intact organism (25, 26). In our study, [Pro ${ }^{11} \mathrm{DAla}^{12}$ ] Ang I did not change LV dP/ $\mathrm{dt}$, despite an increase in LV end diastolic dimension and end diastolic pressure, hemodynamic changes which may be associated with an increased $\mathrm{LV} \mathrm{dP/dt}$, independent of a positive inotropic effect. Moreover, administration of [ $\mathrm{Pro}^{11} \mathrm{DAla}{ }^{12}$ ] Ang I directly into the left coronary artery (to avoid the potential confounding systemic effect of this compound), failed to increase either LV dP/dt or LV shortening. Similar findings with intracoronary Ang II infusions indicate that the lack of response to [Pro ${ }^{11} \mathrm{DAla}^{12}$ ] Ang I was not the result of inadequate myocardial generation of Ang II. Taken together, these data indicate a lack of a measurable positive inotropic effect of Ang II in vivo in primate myocardium. Although we cannot exclude entirely an Ang II-provoked increase in coronary vascular tone, the absence of any change in LV wall thickening when [Pro ${ }^{11-}$ $\mathrm{DAla}^{12}$ ] Ang I was injected directly into the coronary artery makes the possibility of offsetting myocardial ischemia at the doses we used unlikely. A potentially confounding problem is that it may be difficult to dissect direct from indirect adrenergicmediated effects. Although the animals were not sympathetically blocked, the presence of an intact sympathetic nervous system should exaggerate, not attenuate any positive inotropic effect of [Pro ${ }^{11} \mathrm{DAla}^{12}$ ] Ang I. Moreover, in the isolated dog heart, direct inotropic effects of angiotensin II required a dose 60 times greater than the dose that caused sympathetic effects (20). Finally, the inotropic response of isolated muscle is het-
Table V. Chymase-like Activity in Baboon Tissue Homogenates

\begin{tabular}{|c|c|c|c|c|}
\hline Tissues & $\begin{array}{l}2 \text { kidney-1 clip } \\
\text { hypertensive baboons with } \\
\text { LVH chymase-like } \\
\text { activity }(\text { pmol Ang II } \\
\text { generated/min/g } \\
\text { tissue wet weight) }\end{array}$ & $n$ & $\begin{array}{l}\text { Normal baboons } \\
\text { chymase-like activity } \\
\text { (pmol Ang II } \\
\text { generated/min/g } \\
\text { tissue wet weight) }\end{array}$ & $n$ \\
\hline Aorta & $7.1 \pm 4.7$ & 4 & $48.2 \pm 27.4^{*}$ & 5 \\
\hline Diaphragm & $69.3 \pm 55$ & 4 & $59.0 \pm 67.5$ & 5 \\
\hline \multicolumn{5}{|l|}{ Heart } \\
\hline Left ventricle & $26.9 \pm 8.7$ & 4 & $38.4 \pm 11.9$ & 6 \\
\hline Right ventricle & $35.8 \pm 17.0$ & 4 & $69.9 \pm 55.6$ & 6 \\
\hline Intraventricular & & & & \\
\hline septum & $23.5 \pm 15$ & 4 & $40.2 \pm 30.2$ & 5 \\
\hline Lung & $446 \pm 300$ & 4 & $392 \pm 427$ & 5 \\
\hline Skeletal muscle & $39.4 \pm 31.0$ & 4 & $14.6 \pm 3.5$ & 4 \\
\hline Spleen & $12,400 \pm 5,540$ & 4 & $4781 \pm 2467 *$ & 4 \\
\hline
\end{tabular}

$L V H$, left ventricular hypertrophy. ${ }^{\ddagger}$ Chymostatin-blockade conversion of $\left[\mathrm{Pro}^{11} \mathrm{DAla}^{12}\right]$ Ang I to Ang II. All assays were performed in duplicate. ${ }^{*} P<0.05$ vs. clipped.

erogeneous and dependent on the baseline contractile state (23); moreover, coupling of Ang II receptors to inotropic responses in human atrial, but not ventricular myocardium was recently demonstrated (27). Thus, it is possible that dosage, experimental preparation and species and regional differences explain the lack of a positive inotropic effect in our study.

Ang II has complex effects on heart rate. In addition to a direct positive chronotropic effect (19), Ang II increases heart rate by facilitating sympathetic neurohormonal activity and by a central reduction of vagal tone; baroreceptor-mediated slowing of the heart rate in response to Ang II-provoked hypertension opposes these effects (18). Despite a striking elevation of LV systolic pressure, we observed no significant changes in the heart rate. Thus our data suggest a concomitant direct positive chronotropic effect of Ang II in the intact animal. Since our animals were not vagally blocked, a central parasympathetic effect cannot be excluded. Interestingly, heart rate was unchanged when either [Pro ${ }^{11} \mathrm{DAla}^{12}$ ] Ang I or Ang II was infused directly into the left coronary artery; the absence of a chronotropic response to left coronary injection may reflect the right coronary arterial supply of the Ang II receptor-rich sinus node (28) or a dose-related effect.

Ang II effects on diastolic LV function have received scant attention. In isolated myocytes and isolated human atrial trabeculae, Ang II produced a dose-dependent delay in relaxation (23, 29). In isolated, perfused rat hearts, Ang II activation (Ang I infusion) caused a dose-dependent increase in isovolumic LV diastolic pressure in rats with experimental pressure overload left ventricular hypertrophy, but not in sham operated rats (30). In the present study, the time constant of left ventricular relaxation was increased significantly by the large bolus of [Pro ${ }^{11-}$ DAla $^{12}$ ] Ang I, suggesting impaired left ventricular diastolic function. However, this may reflect the large afterload stress produced by Ang II-mediated vasoconstriction. In addition, the small, but significant increase in the time constant of isovolumic $\mathrm{LV}$ relaxation after the $100 \mu \mathrm{g}$ intracoronary bolus may reflect the sensitivity of this index to changes in afterload in anesthetized animals (31). By contrast, low (nonsystemic) doses of 
intracoronary [Pro ${ }^{11} \mathrm{DAla}^{12}$ ] Ang I produced no effect on diastolic function as measured by the time constant of LV relaxation. Thus, our data provide additional evidence that Ang II does not have a significant direct effect on diastolic function in normal myocardium. However, the response to Ang II in hypertrophied primate myocardium is unknown. In aortic banded rats, ACE mRNA expression was increased in the hypertrophied left ventricle compared to sham controls (30). In this regard it is interesting that we found reduced chymase activity in all cardiovascular tissues from hypertensive baboons, although statistical significance was achieved only with aortic tissue.

ACE inhibitors are used widely in the treatment of hypertension and heart failure. An alternative pathway for Ang II production, such as demonstrated in our study, represents a potential means by which tissues could escape from complete ACE inhibition of Ang II production. This may be particularly important in congestive heart failure. In the presence of ACE inhibitors, resulting high levels of Ang I (32) may be shunted to Ang II by chymase. It has been suggested that Ang II may have deleterious direct effects on the failing heart (33). If so, then inhibition of chymase-like activity, in addition to ACE inhibition may prove more beneficial than ACE inhibitors alone in congestive heart failure.

There is also a complex relationship between ACE inhibition, Ang II levels, and clinical effects in systemic hypertension. During chronic therapy with ACE inhibition, plasma Ang II levels return to normal despite continued salutary vascular effects (3). The dissociation between plasma Ang II activity and the efficacy of ACE inhibitors in hypertension may relate in part, to the ability of ACE inhibitors to interact with a variety of substrates such as bradykinin, prostaglandin, substance $P$ and enkephalins (34). In a recent study, ACE inhibition in guinea pigs had an additive beneficial effect on blood pressure over either renin inhibition or Ang II receptor blockade that could not be accounted for by cyclooxygenase inhibition or bradykinin antagonism (35). Thus, chronic suppression of Ang II production by inhibiting tissue chymase-like activity may prove a beneficial adjunct to ACE inhibition in the treatment of systemic hypertension.

In conclusion, intravenous administration of the chymase specific substrate, [Pro ${ }^{11} \mathrm{DAla}^{12}$ ] Ang I to conscious baboons causes a constellation of hemodynamic changes consistent with systemic vasoconstriction and a lack of positive inotropic effect. The high in vitro substrate specificity of [Pro ${ }^{11} \mathrm{DAla}^{12}$ ] Ang I, the reversal of its effects by an Ang II receptor antagonist and the lack of attenuation by captopril, indicate that our findings are due to in vivo conversion to Ang II by chymase, but not ACE. These results suggest that chymase may be involved in the regulation of arterial pressure and may explain the inability of ACE inhibitors to lower Ang II levels chronically. Although chymase-like activity is relatively low in normal and hypertensive hypertrophied primate myocardium compared to lung and spleen, the autocrine and paracrine effects of Ang II generated by this pathway may have significant physiological implications. Whether this ACE independent formation of Ang II by chymase represents a novel cardiac endocrine and/or vascular paracrine function is currently under investigation in our laboratory.

\section{Acknowledgments}

We acknowledge the technical assistance of Gary Flesher, Beverly Bauer, Dennis Wilk, and Tom Friede, and the secretarial support of Norma Burns.
This work was supported in part by National Institute of Health grants HL-33579, HL-44201, and HL-33713 and a Medical School grant from Merck Research Laboratories.

\section{References}

1. Erdos, E. G., and R. A. Skidgel. 1985. Structure and functions of human angiotensin I converting enzyme (kininase II). Biochem. Soc. Trans. 13:42-44. 2. Deboden, A., T. Inagami, and D. Ganten. 1983. Tissue renin. In Hypertension: physiopathology and Treatment. 2nd edition. Edited by J. Genest, O. Kuchel, P. Hamet and M. Cantin, editors. MacGraw-Hill, New York, 194-209.

3. Mento, P. F., and B. M. Wilkes. 1987. Plasma angiotensins and blood pressure during converting enzyme inhibition. Hypertension [Suppl III]:III42III48.

4. Okunishi, H., M. Miyazaki, and N. Toda. 1984. Evidence for a putatively new angiotensin II-generating enzyme in the vascular wall. J. Hypertens. 2:277284.

5. Urata, H., B. Healy, R. W. Stewart, F. M. Bumpus, and A. Husain. 1990 Angiotensin II-forming pathways in normal and failing human hearts. Circ. Res. 66:883-890

6. Urata, H., A. Kinoshita, K. S. Misono, F. M. Bumpus, and A. Husain 1990. Identification of a highly specific chymase as the major angiotensin IIforming enzyme in the human heart. J. Biol. Chem. 265:22348-22357.

7. Urata, H., A. Kinoshita, D. M. Perez, K. S. Misono, F. M. Bumpus, R. M Graham, and A. Husain. 1991. Cloning of the gene and cDNA for human heart chymase. J. Biol. Chem. 266:17173-17179.

8. Urata, H., K. D. Boehm, A. Phillip, A. Kinoshita, J. Gabraovsek, F. M Bumpus, and A. Husain. 1993. Cellular localization and regional distribution of a major angiotensin II-forming chymase in the heart. J. Clin. Invest. 91:12691281

9. Sporn, L. A., V. J. Marder, and D. D. Wagner. 1989. Differing polarity of the constitutive and regulated secretory pathways for von Willebrand Factor in endothelial cells. J. Cell. Biol. 108:1283-1289.

10. Kinoshita, A., H. Urata, F. M. Bumpus, and A. Husain. 1993. Measurement of angiotensin I-converting inhibition (ACE) inhibition in the heart. Circ. Res. 73:51-60.

11. Kinoshita, A., H. Urata, F. M. Bumpus, and A. Husain. 1991. Multiple determinants for the high substrate specificity of an angiotensin II-forming chymase from the human heart. J. Biol. Chem. 266:19192-19197.

12. Husain, A., A. Kinoshita, S. S. Sung, H. Urata, and F. M. Bumpus 1994. Human heart chymase. In The Cardiac Renin-Angiotensin System, eds K Lindpainter and D. Ganten, editors, Futura Publishing Co., Armonk, NY. 309331

13. Applegate, R. J., R. A. Walsh, and R. A. O'Rourke. 1987. Effects of nifedipine on diastolic function during brief periods of flow-limiting ischemia in the conscious dog. Circulation. 76:1409-1421.

14. Walsh, R. A., and R. A. O'Rourke. 1985. Direct and indirect effects of calcium entry blocking agents on isovolumic left ventricular relaxation in conscious dogs. J. Clin. Invest. 75:1426-1434.

15. Weiss, J. L., J. W. Frederickson, and M. L. Weisfeldt. 1976. Hemodynamic determinants of the time course of fall in canine left ventricular pressure. J. Clin Invest. 58:751-760.

16. Starling, M. R., D. G. Montgomery, G. B. J. Mancini, and R. A. Walsh 1987. Load independence of the rate of isovolumic relaxation in man. Circulation. 76:1274-1281.

17. Peach, M. J. 1977. Renin-angiotensin system: biochemistry and mechanisms of action. Physiol. Rev. 57:313-370.

18. Baker, K. M., G. W. Booz, and D. E. Dostal. 1992. Cardiac actions of angiotensin II: role of an intracardiac renin-angiotensin system. Annu. Rev. Physiol. 54:227-241

19. Kobayashi, M., Y. Furukawa, and S. Chiba. 1978. Positive chronotropic and inotropic effects of angiotensin II in the dog heart. Eur. J. Pharmacol. 50:1725 .

20. Farr, W. C., and G. Grupp. 1971. Ganglionic stimulation: mechanism of the positive inotropic and chronotropic effects of angiotensin. J. Pharmacol. Exp. Ther. 177:48-55.

21. Dempsey, P. J., Z. T. McCallum, K. M. Kent, and T. Cooper. 1971. Direct myocardial effects of angiotensin II. Am. J. Physiol. 220(2):477-481.

22. Freer, R., A. Pappano, M. Peach, K. Bing, M. McLean, S. Vogel, and N. Sperelakis. 1976. Mechanisms for the positive inotropic effect of angiotensin II on isolated cardiac muscle. Circ. Res. 39:178-183.

23. Moravec, C. S., M. D. Schluchter, L. Paranandi, B. Czerska, R. W. Stewart, E. Rosenkranz, and M. Bond. 1990. Inotropic effects of angiotensin II on human cardiac muscle in vitro. Circulation. 82:1973-1984.

24. Baker, K. M., and H. A. Singer. 1988. Indentification and characterization of guinea pig antiotensin II ventricular and atrial receptors: coupling to inositol phosphate production. Circ. Res. 62:896-904. 
25. Frank, M. J., M. Nadimi, P. Casanegra, P. Stein, and R. Pekaar. 1970. Effect of angiotensin on myocardial function. Am. J. Physiol. 218(5):1267-1278. 26. Fowler, N. O., and J. C. Holmes. 1964. Coronary and myocardial actions of angiotensin. Circ. Res. 14:191-201.

27. Holubarsch, C., G. Hasenfuss, S. Schmidt-Schweda, A. Knorr, B. Pieske, T. Ruf, R. Fasol, and H. Just. 1993. Angiotensin I and II exert inotropic effects in atrial but not in ventricular human myocardium. An in vitro study under physiological experimental conditions. Circulation 88:1228-1237.

28. Urata, H., B. Healy, R. W. Stewart, F. M. Bumpus, and A. Husain. 1989. Angiotensin II receptors in normal and failing human hearts. J. Clin. Endocrinol. Metab. 69:54-66.

29. Neyses, L., H. Vetter. 1989. Action of atrial natriuretic peptide and angiotensin II on the myocardium: studies in isolated rat ventricular cardiomyocytes. Biochem. Biophys. Res. Commun. 163:1435-1443.

30. Schunkert, H., V. J. Dzau, S. S. Tang, A. T. Hirsch, C. S. Apstein, and B. H. Lorell. 1990. Increased rat cardiac angiotensin converting enzyme activity and mRNA expression in pressure overload left ventricular hypertrophy. J. Clin. Invest. 86:1913-1920.
31. Gaasch, W. H., A. S. Blaustein, C. W. Andrias, R. P. Donahue, and B. Avitall. 1980. Myocardial relaxation: II. Hemodynamic determinants of rate of left ventricular isovolumic pressure decline. Am. J. Physiol. 239 (Heart Circ Physiol 8):H1-H6.

32. Tarazi, R. C., F. M. Fouad, J. K. Ceimo, and E. L. Bravo EL. 1979. Renin, aldosterone and cardiac decompensation: studies with an oral converting enzyme inhibitor in heart failure. Am. J. Cardiol. 44:1013-1018.

33. Hirsch, A. T., C. E. Talsness, H. Schunkert, M. Paul, and V. J. Dzau. 1991. Tissue specific activation of cardiac angiotensin converting enzyme in experimental heart failure. Circ. Res. 69:475-482.

34. Erdos, E. G. 1991. Angiotensin I converting enzyme and the changes in our concepts through the years. Hypertension. 16:363-370.

35. Veniant, M., J. P. Clozel, P. Hess, and W. Fischli. 1992. Effects of angiotensin system blockade in guinea pigs. Hypertension. 19:255-262.

34. Naftilan, A. J., R. J. Pratt, C. S. Eldridge, H. L. Lin, and V. J. Dzau. 1989. Angiotensin II induces c-fos expression in smooth muscle via transcriptional control. Hypertension. 13:706-711. 